\title{
3.2 ENTWURF EINES EMPIRISCHEN ZUGANGS ZUR UNTERSUCHUNG DER SOZIOLOGIE
}

\subsubsection{Das theoretische Sampling}

Zur Grundlegung einer Wissenschaftssoziologie der Soziologie gehört auch, einen adäquaten Analysezugang zu entwickeln. Das Sampling stellt uns vor besondere Herausforderungen, besonders in Hinblick auf den fragmentierten Charakter der Soziologie.

Im Folgenden wird das theoretische Sampling für die Soziologie erläutert. Zunächst erfolgt die Festlegung, dass insbesondere die Art von Soziologie für die Untersuchung interessant ist, die in akademischen Kontexten erzeugt wird, also disziplinäres Wissen und nicht Wissen, das in Think Tanks oder Beratungseinrichtungen entstanden ist. Disziplinen zeichnen sich nach Stichweh (1994) dadurch aus, dass sie

„1) ,typischerweise' über einen hinreichend homogenen Kommunikationszusammenhang von Forschern [verfügen] - eine ,scientific community'; 2) [über] einen Korpus wissenschaftlichen Wissens, der in Lehrbüchern repräsentiert ist [...]; 3.) eine Mehrzahl je gegenwärtig problematischer Fragestellungen; 4) einen ,set' von Forschungsmethoden und paradigmatischen Problemlösungen; 5) eine disziplinenspezifische Karrierestruktur und institutionalisierte Sozialisationsprozesse, die der Selektion und ,Indoktrination“ des Nachwuchses dienen.“ (Stichweh, 1994, S. 17)

Diese Art von Wissen, die Ausbildung des wissenschaftlichen Nachwuchses und das Schaffen eines lehrbaren Korpus wissenschaftlichen Wissens wird in der Regeln in akademischen Institutionen erzeugt, das bedeutet im deutschen Fall in Universitäten und außeruniversitären Forschungseinrichtungen. Die Sampleauswahl orientierte sich dementsprechend an Personen, die in diesen Organisationen tätig waren.

Nach welchen Kriterien diese Personen jedoch ausgewählt wurden, wird im weiteren Verlauf geklärt. Es soll zunächst darum gehen, die Charakteristika der Soziologie als Disziplin zu ermitteln. Dazu werden - in Anlehnung an die Grounded Theory - theoretische Ansätze und empirische Befunde als ,sensibilisierende Konzepte“ (Kelle, 2011, S. 251) für die Fallauswahl herangezogen. So wurden neben theoretischen Aspekten auch verfügbare Daten über die deutsche Soziologie und explorative Interviews zurate gezogen. In einem zweiten Schritt werden unterschiedliche Analysedimensionen für das theoretische Sampling abgeleitet, die eine Fallauswahl und -kontrastierung erlauben. 


\section{Die Soziologie - Eine endlose Karawanserei?}

Bereits die Definition des Fachs Soziologie ist eine Herausforderung für die Operationalisierung der Soziologie in Deutschland. Wer oder was ist Soziologie? Wer gehört dazu, wer nicht? Die Soziologie ist eine hoch ausdifferenzierte und in sich wenig kohärente Disziplin (Whitley, 1984b; Lammers, 1974; Abbott, 2001; Klein, 1996). Wie generalisierungsfähig also sind Aussagen über die Soziologie? Andrew Abbott hebt in seinem Werk „The Chaos of Disciplines“ den heterogenen Charakter der Soziologie hervor. Die Soziologie besteht aus einer Vielzahl unterschiedlicher Fachgebiete und Theorieströmungen:

„The discipline is rather like a caravansary on the Silk Road, filled with all sorts and types of people and beset by bandit gangs of positivists, feminists, interactionists, and Marxists, and even by some larger, far-off states like Economics and the Humanities, all of whom are bent on reducing the place to vassalage. The inhabitants put up with occasional rule by these gangs and pay them tribute when necessary, but when somebody more interesting comes along, they throw off the current overlords with little regret.“ (Abbott, 2001, S. 6)

Den interstitiellen und synthetischen Charakter der Soziologie hebt auch Julie Thompson Klein (1996) hervor. Die Identität der Soziologie sei synthetisch, da die Soziologie sich durch eine Offenheit gegenüber Ideen anderer Disziplinen und durch einen relativ losen Interessenzusammenhang auszeichne. Darüber hinaus sei sie interstitiell, sie versuche also entstehende Lücken mit anderen Sozialwissenschaften ,aufzufüllen“ (Klein, 1996). All dies führe zu verschwommenen Außengrenzen und zu einer eher (zwangsläufig) interdisziplinären Praxis der Soziologie. Was also Soziologie ist und wann sie ihre Grenzen erreicht, wann etwas in den Wissenskanon der Soziologie aufgenommen wird, ist als wenig definiert anzusehen.

Dies stellt die Bündelung der Soziologie in Kategorien vor Herausforderungen. Gesucht sind Strukturierungsmomente der Soziologie, die sich über unterschiedliche Kontexte als stabil erweisen, sowie maximale Unterschiede innerhalb der Disziplin, anhand derer unterschiedliche Definitionen dessen, was Soziologie ausmacht, verhandelt werden.

Für eine theoretische Unterscheidung unterschiedlicher Analyseebenen macht Kaldewey (2013) folgenden Vorschlag: 
Tabelle 6: Dimensionen eines integrativen Wissenschaftsbegriffs ${ }^{11}$

\begin{tabular}{|c|c|}
\hline $\begin{array}{l}\text { Semantische Ebene der Wissen- } \\
\text { schaft }\end{array}$ & $\begin{array}{l}\text { Differenzierung von Diskursen, z.B.: } \\
\text { Methodendiskurse } \\
\text { Theoriediskurse } \\
\text { Autonomiediskurse } \\
\text { Praxisdiskurse }\end{array}$ \\
\hline Operative Ebene der Wissenschaft & $\begin{array}{l}\text { Differenzierung von Subsystemen, } \\
\text { z.B.: } \\
\text { Forschergruppen } \\
\text { Disziplinäre Gemeinschaften } \\
\text { Epistemische Kulturen } \\
\text { Netzwerke, Kollektive, Hybride etc. }\end{array}$ \\
\hline $\begin{array}{l}\text { Organisationale Ebene der Wissen- } \\
\text { schaft }\end{array}$ & $\begin{array}{l}\text { Differenzierung von Organisationen, } \\
\text { z.B.: } \\
\text { Universitäten } \\
\text { Akademien } \\
\text { Forschungsinstitute } \\
\text { Zeitschriften, Verlage }\end{array}$ \\
\hline
\end{tabular}

Diese differenzierungstheoretisch inspirierten Dimensionen lassen sich für die vorliegende Arbeit übersetzen in: 1. Die Ordnung der Soziologie in institutionell-organisationale Kontexte; 2. Die Ordnung der Soziologie in Subdisziplinen; 3. Die Ordnung der Soziologie in verschiedene Diskursgemeinschaften.

Diese Ebenen sind zunächst als Heuristiken zu verstehen, die sich in der (Forschungs-)Praxis überschneiden. Dennoch erlauben diese Heuristiken, mögliche Kategorien der Operationalisierung der Soziologie sinnvoll vorzustrukturieren. Im Sinne der Grounded Theory wurde zunächst neben einer theoretischen Sensibilisierung eine empirische Sensibilisierung des Feldes vorgenommen, um die Kategorien aus dem Material zu generieren und um zu prüfen, ob diese in der empirischen Analyse nützlich sind. Die Kategorien lassen sich gut mit der von Kaldewey vorgeschlagenen Heuristik vereinbaren und werden im Folgenden erläutert.

11 Kaldewey, 2013, S. 152. 
Zunächst wird die unterschiedliche institutionell-organisationale Ordnung der Soziologie in den Blick genommen. Ebenso wird geprüft, inwieweit sich diverse Forschungsfelder, also Subdisziplinen, für das theoretische Sampling der Soziologie eignen. Als Letztes wird die Soziologie als Bündelung verschiedener Schulen, also Diskursgemeinschaften, betrachtet. Am Ende des Kapitels wird ein Schema entworfen, das ein Prinzip zeigt, wie die Fälle gemäß dem theoretischen Sampling für die Soziologie ausgewählt werden können.

\section{Ordnung der Soziologie nach institutionell-organisationalen Kontexten}

Hilfreich für die Auswahl der Untersuchungseinheiten innerhalb des soziologischen Feldes in Deutschland ist das Forschungsrating für Soziologie, das der Wissenschaftsrat zwischen den Jahren 2004 und 2007 durchgeführt hat (Wissenschaftsrat, 2008b; 2008a; Neidhardt, 2006). Das Rating war eine Pilotstudie, die am Fach Soziologie durchgeführt wurde, um die Forschungsleistungen in Universitäten und außeruniversitären Einrichtungen ,vergleichend zu bewerten, um die Einrichtungen bei strategischen Entscheidungen zu unterstützen und durch mehr Transparenz den Wettbewerb zu fördern" (Wissenschaftsrat, 2008b, S. 9). Für die Operationalisierung des Fachs wurde eine Bewertungsgruppe eingesetzt, die aus insgesamt 16 Fachvertretern und -vertreterinnen der Soziologie bestand und deren Mitglieder von der Deutschen Gesellschaft für Soziologie und den großen Forschungsorganisationen vorgeschlagen wurden. Die Soziologie wurde auf zwei Ebenen operationalisiert: die Einteilung der Soziologie in Teilbereiche, um das Fach thematisch von anderen Fächern abzugrenzen, und in unterschiedliche Forschungseinheiten, z.B. in organisationale Einheiten (Universitäten, auBeruniversitäre Forschungseinrichtungen), in denen soziologische Forschung stattfindet. Diese Orte setzen sich zusammen aus staatlichen und ausgewählten privaten Universitäten sowie von Bund und Ländern gemeinsam geförderten auBeruniversitären Forschungseinrichtungen.

Insgesamt reagierten 64 Einrichtungen positiv auf die Anfrage des Wissenschaftsrats und es nahmen 57 Einrichtungen am Rating teil. Interessant ist die hohe interne Fragmentierung der Soziologie auf Ebene der Forschungseinrichtungen. So gab es für Forschungseinrichtungen die Möglichkeit, Subeinheiten festzulegen. Dabei wurden meist sehr kleine Einheiten bestimmt; diese umfassten überwiegend eine Professur und die dazugehörigen Mitarbeiter(innen). Der Wissenschaftsrat erklärte sich diese Differenzierung folgendermaßen: 1. Die Hochschulen würden in der Regel über gewachsene (Instituts- bzw. Seminar-) Strukturen verfügen, die sich an der Lehre orientieren, nicht an der Forschung; eine gemeinsame Identität als Teil einer Einrichtung sei im Fach deshalb häufig 
nicht entwickelt. 2. Soziologische Forschung sei nicht selten in mehreren Fakultäten oder Fachbereichen einer Universität angesiedelt, also in verschiedenen disziplinären Kontexten. Von 20 der 54 teilnehmenden Universitäten wurden Einheiten aus zwei oder mehr Fakultäten/Fachbereichen gemeldet. Der Kontakt der soziologischen Professuren untereinander sei auch deshalb häufig locker, die Bereitschaft, gemeinsam eine Forschungseinheit zu bilden, entsprechend gering. 3. Die Mehrzahl der Forschungseinheiten beschäftige sich nach eigenen Angaben nicht nur mit soziologischen Fragestellungen und wende soziologische Methoden an, sondern forsche multidisziplinär. Ein Viertel der teilnehmenden Einheiten schätzte den Anteil der Soziologie an den eigenen Forschungen auf höchstens 50 Prozent. Dies deute darauf hin, dass die Soziologie an ihren breiten Rändern nur unscharf definiert sei, was die Bildung von größeren Forschungseinheiten erschwert haben dürfte. 4. Außerdem hätten bei der Bestimmung der Forschungseinheiten mit Blick auf die vorgestellten Evaluationsergebnisse auch taktische Überlegungen eine Rolle gespielt (Wissenschaftsrat, 2008b, S. 18).

Wie das Forschungsrating für Soziologie zeigt, ist die Identifikation mit den übergeordneten Forschungseinheiten gering - das wird an der hohen Anzahl extrem kleiner Forschungseinheiten deutlich, die genannt wurden. Die Soziologie kann demnach als eine stark individualisierte und hoch fragmentierte Disziplin bezeichnet werden, in der jeder sein eigenes Süppchen kocht. Auch im Zuge der Erhebung zeigte sich, dass die Interviewpartner sich in der Regel nicht ausschließlich mit einer einzigen Forschungseinrichtung identifizierten. Zudem zeichneten sie sich aufgrund befristeter Stellen und bestimmter Karrierevorstellungen durch eine relativ hohe Mobilität aus und wurden deshalb meist in mehreren Einrichtungen beruflich sozialisiert. Die institutionelle Verortung wurde in einigen Interviews durchaus thematisiert, insbesondere, wenn es sich um eine langjährige Zugehörigkeit zu einem Institut oder einer außeruniversitären Forschungseinrichtung handelte. Dennoch erschien es für eine Kontrastierung nicht sinnvoll, die Fälle ausschließlich anhand ortsgebundener Kategorien zu differenzieren, wenngleich sie in der Analyse Berücksichtigung fanden. Eine solche Differenzierung war dann von besonderem Interesse, wenn sie von den Interviewten selbst benannt wurde.

Diese Befunde entsprechen im Wesentlichen auch Aussagen der Organisations- und Professionssoziologie, die wissenschaftliche Organisationen als „specific organizations“ bezeichnen (Musselin, 2007). Professionen zeichnen sich durch relativ hohe Freiheitsgrade aus und sind nicht immer mit Organisationszielen kongruent. Im klassischen Sinn sind Professionen „sich selbst verwaltende, in Berufsausbildung und Berufsausübung relativ autonome, durch kollegiale Binnenkontrolle geprägte, korporative Gebilde“ (Pfadenhauer et al., 2010, 
S. 364), ihre Aktivitäten sind „legitimately guided by external interests, values, norms and standards, rather than by an internally generated organizational policy“" (Brunsson et al., 2000, S. 734). In diesem Sinn sind wissenschaftliche Organisationen als „incomplete“ (Brunsson et al., 2000, S. 734), also unvollständig zu begreifen.

\section{Ordnung der Soziologie nach Subdisziplinen}

Die Wissenschaftsforschung geht von der Annahme aus, dass sich die moderne Wissenschaft zunehmend spezialisiert: „Specialization is a hallmark of modern science." (Chubin, 1976, S. 448) Dies zeigt sich zunächst in der Ausbildung diverser Disziplinen und in einem weiteren Schritt in der Ausbildung unterschiedlicher Subeinheiten innerhalb der Disziplinen und an deren Rändern. In der Literatur finden sich zahlreiche Bündelungsversuche, die Subeinheiten der Wissenschaften zu fassen und zu charakterisieren suchen. Chubin (1976) sieht den Unterschied zwischen Disziplinen und ihren Subeinheiten darin, dass Disziplinen und ihre Organisation in bürokratischen Strukturen (z.B. akademische Departments) mit dem Training und der Zertifizierung von Wissenschaftlern und Wissenschaftlerinnen beschäftigt sind. Forschung, die den Anforderungen der „Zertifizierung" der Disziplin entspricht, trägt jedoch selten zum Wissensfortschritt der gesamten Disziplin bei. Vielmehr findet dieser eher in thematischen Subfeldern statt:

„In short, disciplines form the teaching domain of science, while smaller intellectual units (nestled within and between disciplines) comprise the research domain. Within the sociology of science, these units have been termed ,scientific specialties“" (Chubin, 1976, S. 448)

Die Soziologie zeichnet sich durch eine hohe Fragmentierung und somit auch sehr disperse Subfelder aus, die - je nach Ausrichtung - Nähe zu anderen Disziplinen bzw. interdisziplinären Charakter aufweisen. ${ }^{12}$ Diese specialties werden als „microenvironments for research“ (Hagstrom, 1970, S. 93) charakterisiert. „A discipline is composed of clusters of specialties that form the microenvironments where research and communication take place." (Klein, 1996, S. 42) Die soziale Dimension der microenvironments drückt sich aus in einer gezielten

12 So weisen beispielsweise die Forschungsfelder Bildungs- und Jugendsoziologie eine große Nähe zur Psychologie und Erziehungswissenschaft auf oder die Frauen- und Geschlechterforschung zu den interdisziplinär angelegten Gender Studies, die sich aus Sozial-, Kultur- und Geisteswissenschaften zusammensetzen. 
Kommunikation mit einer Gruppe von Individuen, die in der Regel kleiner ist als eine Disziplin. Sie streben innerhalb einer Disziplin Status sowie Ressourcen im Feld an und besetzen wichtige Positionen in professionellen Vereinigungen, Journals und Graduiertenprogrammen (Klein, 1996). Innovationen oder Veränderungen werden meist durch Akteure angeregt, die neu in die Subdisziplin eintreten. So zeichnet sich ein neues Subfeld auch durch die Fluidität ihrer Mitglieder aus bzw. Mitglieder einer Disziplin können auch in mehreren Subdisziplinen präsent sein (Klein, 1996). Aus systemtheoretischer Sicht sind solche Subeinheiten in Spezialgebiete oder Problemfelder als fortschreitender Prozess der Differenzierung innerhalb der Disziplinen zu begreifen (Stichweh, 1994). Diese Spezialgebiete siedeln sich entweder innerhalb oder zwischen Disziplinen an. Sie sind in Anlehnung an Polanyi gemäß einem „Fischschuppenmodell[...]“ (Stichweh, 1994, S. 40) zu verstehen, denn sie decken unterschiedliche Problemfelder $\mathrm{ab}$ und überlappen einander.

Nichtsdestoweniger sind Definitionen und Formen der Erfassung von „specialisms" relativ dispers und variabel. ${ }^{13}$ Klassifikationen finden auf struktureller Ebene statt, beispielsweise mithilfe bibliometrischer Analysen: Die Anzahl der Publikationen in einem bestimmten Fachgebiet gibt Aufschluss über die Entwicklung einer Subdisziplin, und Zitationen werden als formale Manifestationen der Kommunikation zwischen zwei Wissenschaftlern angesehen (Solla Price, D. J., de, 1965). Insbesondere Co-Zitationen weisen auf einen zunehmend kohärenten Wissenskanon hin (Chubin, 1976). Mit der Formierung von Subfeldern ist deshalb immer auch eine Verdichtung der Kommunikation verbunden. Mullins und Mullins (1973) stellen drei Stufen der Gruppenentwicklung fest: Gruppen bis zu 40 Personen in einem Netzwerk, 7 bis 25 in einem Cluster und 20 bis 100 in einer Subdisziplin.

Kadushin (1966; 1968) bezeichnet eine solche Verdichtung als „social circles". Die Enden dieser Kreise sind lose und schwer definierbar. Jedes Mitglied ist sich in der Regel mancher, jedoch nicht aller Mitglieder bewusst. Meist sind diese auch noch räumlich getrennt und standen oft noch nie in persönlichem Kontakt. Der indirekte Kontakt ist ein wichtiger Aspekt sozialer Kreise. Sie haben auch keine formale Führungsposition, allerdings existieren in den Kreisen zentrale Personen (Crane, 1972). Diese bezeichnet Crane (1972) als ,invisible colleges“. Sie sind hilfreich für die Herstellung einer Gruppensolidarität und ei-

13 Für einen Überblick siehe beispielsweise Chubin, 1976 oder Becher et al., 2001, S. 58 ff. 
ner gemeinsamen Moral, die laut Crane die Mitglieder der Zirkel motiviert (Crane, 1972 , S. 138 f.). ${ }^{14}$

Dieser kurze Einblick in theoretische Konzepte, die specialisms oder Subdisziplinen spezifizieren, macht deutlich, dass Subdisziplinen zwar einen wichtigen Teil disziplinärer Entwicklung darstellen, jedoch schwer empirisch zu operationalisieren sind, weil sie so dispers sind und sich an den Rändern teilweise überlappen. Auch in der Empirie zeigte sich, dass eine Einteilung der Soziologie in Subdisziplinen schwierig ist. Als Veranschaulichung soll zunächst wieder das Forschungsrating der Soziologie heranzogen werden: Hier wurden insgesamt 25 Subeinheiten für die Soziologie definiert, welche sich im Großen und Ganzen an den Sektionen der DGS orientieren. Auch diese Differenzierung ist auf empirischer Ebene sehr problematisch. So waren die ersten Interviews wenig erfolgversprechend. Die meisten Interviewten rechneten sich unterschiedlichen Forschungsfeldern zu (so lag meist der Schwerpunkt auf einer oder mehreren Methoden, unterschiedlichen Theorierichtungen und Thematiken), manche stellten gar keinen Zusammenhang zu den Sektionen der DGS her, da sie nicht in der DGS aktiv waren. Besonders schwierig war die Zuordnung zu Subdisziplinen, wenn es beispielsweise einen Wechsel an einen anderen Lehrstuhl oder einen personellen Wechsel in einer Forschungseinheit gegeben hatte, weil Forschende dann entweder selbst ihren Forschungsschwerpunkt oder mit ihrem Professor den Standort ändern. Im Forschungsrating der Soziologie ließ sich zudem nachweisen, dass zwar eine Unterscheidung in unterschiedliche Fachbereiche der Disziplin und die Abgrenzung zu anderen Bereichen - Publizistik und allgemeine Kommunikationsforschung, Sozialgeschichte, pädagogische Sozialkunde, Sozialpsychologie und Sozialpädagogik (Wissenschaftsrat, 2008b) - sinnvoll und vonnöten war, um das Fach Soziologie zu definieren. Jedoch spielte die oben aufgeführte definitorische Zuordnung bei der Darstellung der einzelnen Forschungseinheiten nur noch eine marginale Rolle, da diese sich meist auf organisatorischer Ebene auf vorhandene Bezeichnungen der Lehrstühle und Abteilun-

14 Whitley unterscheidet zwischen „specialities“ und „research areas“ (Whitley, 1976, S. 472). Für ihn unterscheiden sich die beiden darin, dass Mitglieder einer „research area“ über die Spezifizierung bestimmter Problemdefinition und geeigneter Techniken, um diese zu bearbeiten, übereingekommen sind. „Specialities“ hingegen ,are focussed on explanatory models and definitions of the phenomena under consideration. Membership of specialties implies commitment to particular types of accounts and preferred ways of formulating the underlying object of concern“ (Whitley, 1976, S. 473). Im Gegensatz zu Crane (1972) unterstellt er, dass keiner dieser Typen zwangsläufig einem Solidaritätsmodell unterworfen ist. 
gen beriefen. Ebenso sind gerade auf Ebene der unterschiedlichen Fachgebiete die Übergänge zu anderen Disziplinen fließend, etwa zur Pädagogik oder Psychologie. Darauf lässt auch die Aussage des Wissenschaftsrats schließen, die Soziologie sei an ihren Rändern schlecht definiert und ein Teil der in Deutschland praktizierten Soziologie sei interdisziplinär. Dies erschwert eine empirische Auswahl von Soziologinnen und Soziologen, die sich an Forschungsbereichen und Subdisziplinen orientiert. Obwohl die thematische Zuordnung eine Kategorie für die Auswertung darstellte, kam im Verlauf der weiteren empirischen Konzeptionierung die Frage auf, inwieweit subdisziplinäre Ausdifferenzierungen auf der Ebene der Fallkontrastierung als Merkmal der minimalen und maximalen Unterscheidung dienen können.

\section{Die Ordnung der Soziologie in verschiedene Diskursgemeinschaften}

Eine weitere Möglichkeit, die soziale Organisation der Wissenschaft zusammenzufassen, ist, sie in sogenannte Diskursgemeinschaften einzuteilen. Der Begriff der Diskursgemeinschaften wird in diesem Kontext mit der Entstehung diverser Schulen in Zusammenhang gebracht:

„A school is characterized by the uncritical acceptance on the part of disciples of a leader's idea system. It rejects external influence and validation of its work. By creating a journal of its own, such a group can bypass the criticism of referees from other areas. [...] In extreme cases [...] such groups forgo the attempt to obtain empirical verification for their ideas altogether." (Crane, 1972, S. 87 f.)

Mit der Entstehung unterschiedlicher Schulen (die sich in diversen Methodenoder Theoriediskursen bündeln lassen) ist auch ein jeweils spezifisches Forschungsparadigma verbunden (Crane, 1972; Kuhn, 2003[1962]). Der Unterschied zwischen einer Schule und einer Solidaritätsgruppe ist schwer zu fassen. In beiden Fällen spielt eine starke Leitfigur eine zentrale Rolle. In ihrem Werk „Theories and theory groups in contemporary American Sociology“ erklären Mullins und Mullins (1973) die Emergenz unterschiedlicher Theorien in der Amerikanischen Soziologie. Sie rekurrieren auf vier analytische Ansätze, soziologisches Gedankengut zu bündeln: „,core concepts“; „biography“; , ,intellectual history“ und "schools of theory“ (Tabelle 7). 
Tabelle 7: Analytische Ansätze der Bündelung soziologischen Gedankenguts ${ }^{15}$

\begin{tabular}{|l|l|l|}
\hline & Ideas & Persons \\
\hline Unit & Core concepts & Biographies \\
\hline System & Intellectual history & $\begin{array}{l}\text { Schools of social } \\
\text { thought }\end{array}$ \\
\hline
\end{tabular}

Sie definieren Theorieschulen folgendermaßen:

„[They are] certain similar theories [that] are written in roughly the same period of time by a limited group of authors. [...] Attempts to make sense of these patterns of rising and falling have resulted in the study of social groups or schools of thought. [...] School analysis is largely the product of a socially and psychologically informed philosophy of science which emphasizes the importance of early training and the acquisition of paradigms." (Mullins et al., 1973, S. 11)

Wie strategisch wichtig unterschiedliche Schulen für die Entwicklung der Soziologie sind, wird besonders hervorgehoben (Lepenies, 1981c; Mullins et al., 1973). Nach Lepenies (1981a) ist diese Art der Organisation von Soziologie Teil ihrer kognitiven Identität. Diese zeichnet sich durch „,soziologische Orientierungen, Paradigmen, Problemstellungen und Forschungswerkzeuge[...]“ (Lepenies, 1981a, S. I) aus. Die kognitive Identität ist immer eng verschränkt mit der sozialen Identität, also jenen „Institutionalisierungsprozesse[n], durch die das Fach versucht[...], sich organisatorisch zu stabilisieren“ (Lepenies, 1981a, S. I). Erst durch eine Stabilisierung ist die historische Nachhaltigkeit gesichert. Eine historische Identität liegt demnach vor, wenn es den Mitgliedern gelingt, „eine disziplinäre Vergangenheit zu rekonstruieren, auf die sich im Prinzip alle Mitglieder der soziologischen Wissenschaftlergemeinschaft berufen“ (Lepenies, 1981a, S. I) können. Die Rolle identitätsstiftender Symbole stellen Becher und Trowler in ihrem Ansatz der ,academic tribes and territories“ heraus (Becher et al., 2001). Sie beschreiben in Anlehnung an den Anthropologen Clifford Geertz Disziplinen als einen ,academic tribe“, zu dem die Existenz von Idolen (z.B. Max Weber, Karl Marx, Émile Durkheim), Artefakten und eine eigene Sprache als Distinktionsmerkmal gehören. Der professionellen Sprache und der damit verbundenen Literatur räumen sie einen großen Stellenwert bei der Entstehung einer disziplinären Identität ein. Ebenso haben explizit kulturelle Elemente - ,their

15 Mullins et al., 1973, S. 7. 
traditions, customs and practices, transmitted knowledge, beliefs, morals and rules of conduct, as well as their linguistic and symbolic forms of communication and the meanings they share"(Becher et al., 2001, S. 24) - eine wichtige integrierende Funktion. Mit diesem ethnologischen Zugang entwerfen Becher und Trowler eine Disziplin als eine Art Stamm, der über eigene Mythen, Bilder anderer Disziplinen (im Sinne von einer Konstruktion der „Anderen“) und eigene Anerkennungs- und Sozialisationsinstanzen verfügt (Becher et al., 2001).

Wer also einer Disziplin angehören möchte, muss sich zunächst im System bewähren und die Identitätsmechanismen dieser Disziplin anerkennen. Hinsichtlich der Bewährungsmechanismen in wissenschaftlichen Feldern argumentiert Bourdieu strukturkonservativ: Die Logik des Feldes, ihre „,illusio“, entfaltet ihre Wirkung, um die Spiele mitzuspielen und die Kämpfe mit den Waffen auszutragen, die in diesem Feld eine Rolle spielen. Bewaffnen kann man sich mithilfe unterschiedlicher Kapitalarten. Die erste Art des Kapitals beruht laut Bourdieu auf ,weltlicher Macht“, also strategisch günstigen Positionen im universitären System. Diese Positionen bestimmen über die Produktion und Reproduktion wissenschaftlicher Stellen. Die zweite Art des Kapitals bezeichnet er als „rein wissenschaftlich“ und verbindet dies mit „persönlichem Prestige“ der Person, das auf der Anerkennung der ,invisible colleges“ beruht (Bourdieu, 1998). Andererseits ist das Fortkommen in einem Feld abhängig von dem Habitus der Forschenden. Hier spielen natürlich - wie für Bourdieu kennzeichnend - die Dispositionen mit, die die Einzelnen aus ihrer Primärsozialisation mitbringen. Ebenso ist die Sekundärsozialisation (und hier schließt sich der Kreis zu Becher und Trowler) in dem jeweiligen Feld von großer Bedeutung:

„Diejenigen, die fernab des Feldes, dem sie angehören, ihre Einstellungen erworben haben, andere, als sie das Feld erfordert, laufen deshalb Gefahr, immer verspätet, fehl am Platz, am falschen Platz zu sein, sich unwohl in ihrer Haut zu fühlen, gegen die Schwerkraft, gegen die Zeit anrennen zu müssen, mit all den Folgen, die sich wohl lebhaft ausmalen lassen.“ (Bourdieu, 1998, S. 25)

Auch Schulen sind dadurch gekennzeichnet, dass sie eine Identität der Zugehörigkeit zu einer Diskursgemeinschaft ausbilden, die über eigene Sozialisationsinstanzen verfügt. Szacki (1981) meint, dass als Schule zunächst jede Art von Gruppe von Soziolog(inn)en gelten kann, die zu einem ähnlichen Thema mit einem ähnlichen Zugang innerhalb einer Universität oder eines Instituts arbeiten. Das Kontrastbeispiel hierzu wären aus seiner Sicht sogenannte „nationale“ Schulen, die alle Soziologinnen umfassen, die innerhalb eines determinierten Kulturraums arbeiten (Szacki, 1981). Meist werden Begriffe wie „soziologischer Theo- 
rietyp“, „Doktrin“, „Paradigma“ oder „Gesamtsystem“ mit dem Schulenbegriff gleichgestellt. Umso wichtiger ist die Eingrenzung dessen, was als Schule gelten kann und welche Ausprägungen sie findet. Szacki unterscheidet in Zusammenhang mit dem Schulenbegriff der Soziologie drei Arten von Bedeutungsebenen: die institutionelle, die psychologische und die typologische. Die institutionelle Ebene beschreibt „Interessen, Annahmen, Behauptungen usw., die einer gegebenen Gruppe von Wissenschaftlern gemeinsam sind, innerhalb eines institutionellen Rahmens ausgebildet und abgesichert werden" (Szacki, 1981, S. 18). Auch Krohn und Küppers (1989) zeigen, wie auf institutioneller Ebene eine Art Identitätsbildung stattfinden kann: auf der Ebene der Forschungsgruppen. Sie argumentieren, dass Forschungsabteilungen eigene „Überzeugungen“ (Gruppenmatrix) und Identitäten ausbilden, und benennen vier Ebenen, die Bestandteil einer Gruppenmatrix sind: die kognitive, die soziale, die emotionale und die reflexive Ebene. Weil die Mitglieder einer Forschungsgruppe miteinander interagieren, wird 1. ein gemeinsamer Denkstil ausgebildet (kognitive Ebene); 2. das Gruppenverhalten stabilisiert (soziale Ebene); 3. das Engagement in der Gruppe verstärkt, desto mehr, je deutlicher gemeinsame Normen und Werte geteilt wurden und je reputationsträchtiger die Gruppe von außen erscheint (emotionale Ebene); 4. eine Gruppenidentität ausgebildet, indem ein eigenes Selbst- und Fremdbild entwickelt wird und sich die Gruppe so von anderen Gruppen abgrenzt (reflexive Ebene). Die Gruppe charakterisiert sich demzufolge durch gemeinsame Überzeugungen. Diese sind einerseits theoretischen und methodologischen Charakters. Andererseits zeichnen sie sich aus durch spezifische Annahmen ihrer inner- und außerwissenschaftlichen Relevanz. Sie schaffen Projektpläne, die als koordinierende Instanz dienen (Krohn et al., 1989, S. 34 ff.). Diese Aspekte können dementsprechend auch unter Punkt 2.3.1.2 eine Rolle spielen, wenn sich, wie bereits aufgeführt, die Mitglieder lange genug in einer Forschungsgruppe befinden.

Jedoch ist daran nicht zwingend das Entstehen einer Schule geknüpft. In diesem Sinn führt Szacki (1981) das zweite Kriterium auf, das auf die psychologische Ebene hinweist, also die Ebene des Zusammenhalts einer Gruppe. Hier geht es stärker um die Bedeutung einer Schule als eine Bezugsgruppe, mit der sich unterschiedliche Mitglieder identifizieren, die jedoch als ,invisible colleges“ (Crane, 1972, S. 49) nicht persönlich präsent oder einander bekannt sein müssen. Den Identitätscharakter hebt auch Tiryakian (1981) in seiner Analyse der Rolle hervor, die Schulen für die Entstehung der Soziologie spielen. Er versteht unter einer Schule eine „tatsächlich bestehende Gruppe von Intellektuellen, eine kleine Gemeinschaft von Personen, deren Herkunft und Prägung zeitlich und örtlich lokalisierbar sind“" (Tiryakian, 1981, S. 39). Schulen haben in ihrem Entstehungs- 
stadium meist religiösen Charakter und ihre Mitglieder haben gewöhnlich intellektuellen Missionswillen. Schulen suchen für ihre Implementierung Wege, innerhalb der Profession anerkannt zu werden. Das bedeutet auch, dass sie institutionelle Standorte und entsprechende Publikationsorgane schaffen oder Lehrbücher verfassen. Meist verkündet ihr Gründer eine grundlegende Auffassung der Wirklichkeit, die die Mitglieder auf ihren Untersuchungsgegenstand anwenden sollen. Im Umkehrschluss tendieren die Mitglieder dazu, diese Grundeinstellungen empirisch abzusichern. Es ist für das Überleben einer Schule deshalb von eminenter Wichtigkeit, geeignete Popularisierer und Interpreten der „Botschaft“ zu finden und so größeren Einfluss auf die Disziplin auszuüben. In diesem Sinn konstruiert Tiryakian unterschiedliche Rollentypen, die an der Entstehung von Schulen beteiligt sind: einen Führer und dessen Gefolgschaft, eine kleine Anzahl bedeutender Bekehrter, die der gleichen Generation angehören wie ihr Führer. Ebenso muss eine Gruppe jüngerer Schüler entstehen, die von seinem Geist bereits zu einem frühen Zeitpunkt geprägt wurden und später neue Schüler ausbilden können. Sie ziehen ihre Legimitation aus dem Ausbildungsstatus, den sie beim Gründer der Schule erworben haben. Zu einem späteren Zeitpunkt bekleiden sie selbst Schlüsselpositionen in anderen Institutionen, werben Forschungsgelder ein oder besetzen Ämter bei Berufsverbänden. Eine weitere Rolle kann in der des „Helfers“ bestehen. Ein Helfer ist ein treuer Fußsoldat, der der Schule eine Bühne zur Selbstdarstellung bietet. Dies kann ein Zeitschriftenherausgeber, ein Beamter im Staatsdienst oder der Geschäftsführer einer Stiftung sein (Tiryakian, 1981, S. 45). Ein Patron kann auf die gleiche Weise agieren, indem er der Schule gegenüber positiv eingestellt ist. Erfolgreich ist eine Schule nach Tiryakian, wenn sie sich länger als eine Generation in der Disziplin halten kann. Ebenso bedeutsam ist der dynamische Aspekt einer Schule, also dass sich ihre Denkmuster über die Zeit hinweg verändern können. Diese stark kultursoziologisch geprägte Ansicht der sozialen Organisation einer Schule schließt an die Annahmen von Becher und Trowler (2001) an, wie sie bereits weiter oben skizziert wurden.

Das typologische Verständnis einer Schule hingegen orientiert sich weder an der psychologischen noch an der institutionellen Ebene. Es ordnet Theoriegruppen mit ähnlichen Charakteristika einer Schule zu, die auch mit Begriffen wie „Modellen“, „Perspektiven“, oder „Orientierungen“" umschrieben werden (Szacki, 1981, S. 22).

Wichtig ist bei der Betrachtung von Diskursgemeinschaften als Schulen, dass diese idealtypisch zu verstehen sind, sich nicht zwangsläufig aneinander anschließen und die Soziologie nicht lückenlos abbilden. Im Allgemeinen wird an dem Schulenkonzept kritisiert, dass diese Klassifikationen leicht zu Stereotypi- 
sierungen wie „der Marxismus“ oder „der Funktionalismus“ führen können (Szacki, 1981).

In den explorativen Interviews zeigte sich, dass sich die Interviewpartner besonders auf Diskursgemeinschaften wie Schulen, Denkgemeinschaften, Theoriegruppen bezogen, weshalb sich das theoretische Sampling im weiteren Verlauf der Interviewanalyse insbesondere an der theoretischen wie methodologischen Ausrichtung der Interviewpartner orientiert.

\section{Zwischenfazit}

Gesucht sind Strukturierungsmomente der Soziologie, die sich über den organisationalen Kontext wie auch den thematischen Kontext als stabil erweisen und geeignet sind, um eine maximale und minimale Kontrastierung der Fälle herzustellen. Gezeigt wurde, dass Bündelungsversuche hinsichtlich der Darstellung der Soziologie um die Definition von Subeinheiten kreisen, was sich insbesondere aus der fragmentierten Struktur der Disziplin ableitet. Vor allem die deutsche Soziologie wird, im Gegensatz zu anderen nationalen Soziologien wie z.B. der französischen, als besonders dispers beschrieben (Lepenies, 1981c).

Auch die Ergebnisse des Forschungsratings der Soziologie verdeutlichen, dass sich die Disziplin in Deutschland durch eine hohe Fragmentierung auszeichnet. Forschungseinheiten wurden weitestgehend aus einer Professur gebildet, die Kontakte zwischen den Lehrstühlen werden das deutsche System betreffend als relativ gering betrachtet. Die Ergebnisse der ersten explorativen Interviews zeigen, dass zwar die institutionelle Struktur und Anbindung nicht zu vernachlässigen ist, jedoch ebenso die räumliche Mobilität der Soziolog(inn)en eine Rolle spielt.

Zudem ging aus den explorativen Interviews hervor, dass eine Einteilung in Forschungsfelder, die sich an die Sektionen der DGS anlehnt, keine sinnvolle Strukturierung erlaubt. Das ergibt sich daraus, dass sich die Mehrheit der Befragten mehr als einem Forschungsfeld zuordnet und dass im Verlauf der wissenschaftlichen Karriere häufig Wechsel zu anderen Forschungsfeldern vorkommen. Als erfolgversprechend für eine Strukturierung hat sich die kognitive Affinität erwiesen, weil sich Soziolog(inn)en einer bestimmten Denkrichtung zuordneten.

Aus den Ausführungen in den vorhergehenden Kapiteln lassen sich deshalb zunächst drei Kriterien für Strukturierungsmomente in der Soziologie ableiten: die strukturelle Ähnlichkeit, die kognitive Affinität und die historische Regelmäßigkeit (Abbildung 2). 


\section{Abbildung 2: Strukturierungsmomente der Soziologie}

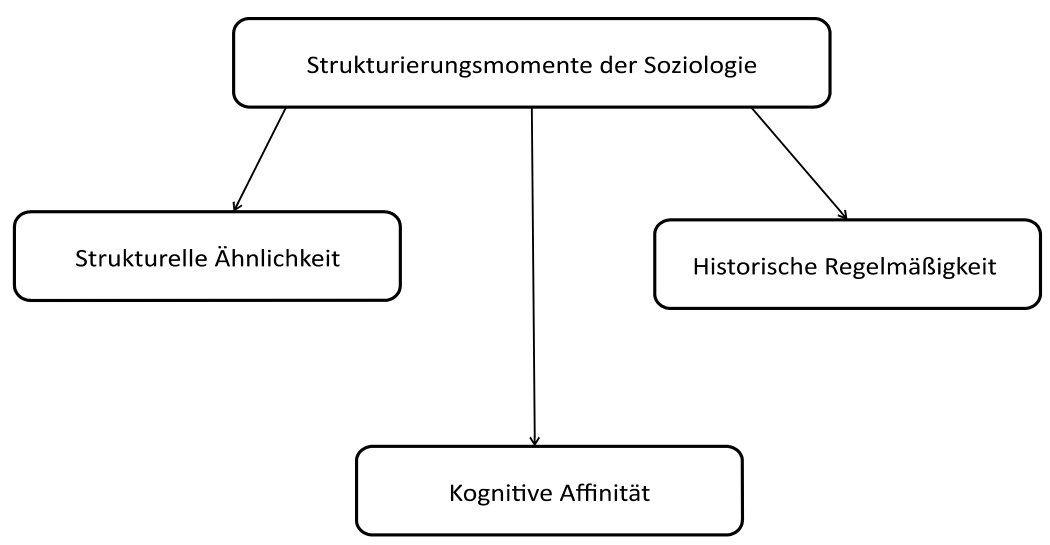

Die Soziologie erweist sich als strukturell ähnlich beschaffen, da ihre Teilbereiche über übergreifende Merkmale wie einen homogenen Kommunikationszusammenhang zwischen Forschenden sowie ein Korpus wissenschaftlichen Wissens, ein Set an bestimmten Forschungsmethoden und eine disziplinenspezifische Karrierestruktur und institutionalisierte Sozialisationsprozesse verfügen (Stichweh, 1994, S. 17). Sie ist in Form diverser Forschungseinrichtungen und -institute langfristig institutionalisiert (Schäfers, 1995; Lepenies, 1981a; 1981b). Dies reicht jedoch zu einer Fallauswahl und Fallkontrastierung nicht aus, sondern bestimmt zunächst die Begrenzung der Soziologie auf einen akademischen Rahmen. Es hat sich in den vorhergehenden Ausführungen gezeigt, dass insbesondere eine kognitive Affinität in Form von Schulen oder Denktraditionen Bindungskräfte entfalten kann. Lepenies (1981a) hat dies mit der kognitiven Identität der Soziologie benannt. Für eine Fallauswahl und Fallkontrastierung muss jedoch im weiteren Verlauf bestimmt werden, welche Art von Schulen, Diskursgemeinschaften oder Denktraditionen sich für die Analyse eignet. Als dritter Punkt soll zudem die historische Regelmäßigkeit hervorgehoben werden. So ist die Analyse einer Debatte besonders dann interessant, wenn sie nicht singulär vorkommt, sondern sich im Verlauf der Zeit wiederholt. Sie hat sich über einen längeren Zeitraum in ihrer Fragestellung als stabil erwiesen und eine „historische Identität“ (Lepenies, 1981a, S. I) entwickelt. Abbott spricht in diesem Zusammenhang von fraktalen Zyklen (Abbott, 2001, S. 22), in denen sich innerdisziplinäre Debatten in unterschiedlichen Generationen zu einem gewissen Grad wiederholen und aufeinander Bezug nehmen. Diese Debatten bilden Dichotomien aus und stehen einander damit gegenüber. Sein Beispiel für eine solche Debatte 
ist die zwischen „Social Structure“ und „Culture“ (Abbott, 2001, S. 13). Wie wir bereits in Kapitel 2 sehen konnten, hat auch die Debatte um die Grenzen zwischen der Soziologie und ihrem Untersuchungsgegenstand eine historische Regelmäßigkeit, da sie sich in unterschiedlichen Epochen in diversen Varianten wiederholt und bis zum heutigen Tag nicht abgeschlossen wurde.

Wie jedoch werden die einzelnen Fälle ausgewählt, in denen sich Sozio$\log (\mathrm{inn})$ en zu dem Verhältnis von Soziologie und ihrem Untersuchungsgegenstand äußern? Wie bereits beschrieben, erweisen sich Diskursgemeinschaften in diesem Zusammenhang als interessante Alternative, da sie institutionelle, historische und identitäre Aspekte miteinander verbinden. Sie wurden in dem hier vorliegenden Fall als soziologische Schulen, Denk- oder Theorietraditionen spezifiziert. Hier muss vorab konstatiert werden, dass eine solche Unterscheidung zunächst nur heuristisch und idealtypisch vorgenommen werden kann. Nichtsdestoweniger ist der Ansatz fruchtbar, epistemologische Grundorientierungen der Forschenden in Form einer Zuordnung zu Theoriegruppen oder Schulen in die empirische Konzeption der Arbeit aufzunehmen. Diese sind bisweilen, jedoch nicht zwingend mit Orten verknüpft, an denen Soziologie betrieben wird (z.B. Systemtheorie mit der Universität Bielefeld). Dieser Ansatz bietet also einerseits die notwendige Offenheit für die weitere Auswahl von Interviewpartnern, vereint jedoch trotz allem wichtige Aspekte für die weitere Analyse. Eine Form der groben Strukturierung und Kontrastierung unterschiedlicher Schulen in der Soziologie ist zunächst die Unterscheidung zwischen Theorie- und Empirieschulen (Kaldewey, 2013). ${ }^{16}$ Abbott dichotomisiert hier einerseits in Methodendiskurse, die er ,fractal distinctions“ (Abbott, 2001, S. 10) nennt und die quantitativ oder qualitativ ausgeprägt sind und unterschiedlichen Paradigmen folgen, grob gesagt, dem positivistischen und dem interpretativen Paradigma. ${ }^{17}$ In Hinblick auf die Theorieschulen sei auf das historische Kapitel verwiesen, in der sich die kritische Theorie (Habermas) und die Systemtheorie (Luhmann) gegenüberstanden, die beide innerhalb der deutschen Soziologie viel betrieben werden.

16 Kaldewey jedoch nennt diese in Anlehnung an Luhmann Methoden- und Theoriediskurse (Kaldewey, 2013, S. 148).

17 Abbott (2001) hat auch hier berechtigterweise noch einmal differenziert, und zwar in quantitative fractal distinctions, die eher interpretativ und eher positivistisch geprägt sind, und qualitative fractal distinctions, die interpretativ wie auch positivistisch geprägt sein können. Für die Auswahl des Samples sei es jedoch bei der groben Unterscheidung quantitativ und qualitativ belassen, da eine genauere Überprüfung dessen, was die einzelnen Interviewpartner mit den Methoden verbinden, erst durch die Analyse der Interviews zum Vorschein kommt. 
Ein theoretisches Sampling kann deshalb folgendermaßen aussehen (Abbildung 3):

\section{Abbildung 3: Theoretisches Sampling der Soziologie}

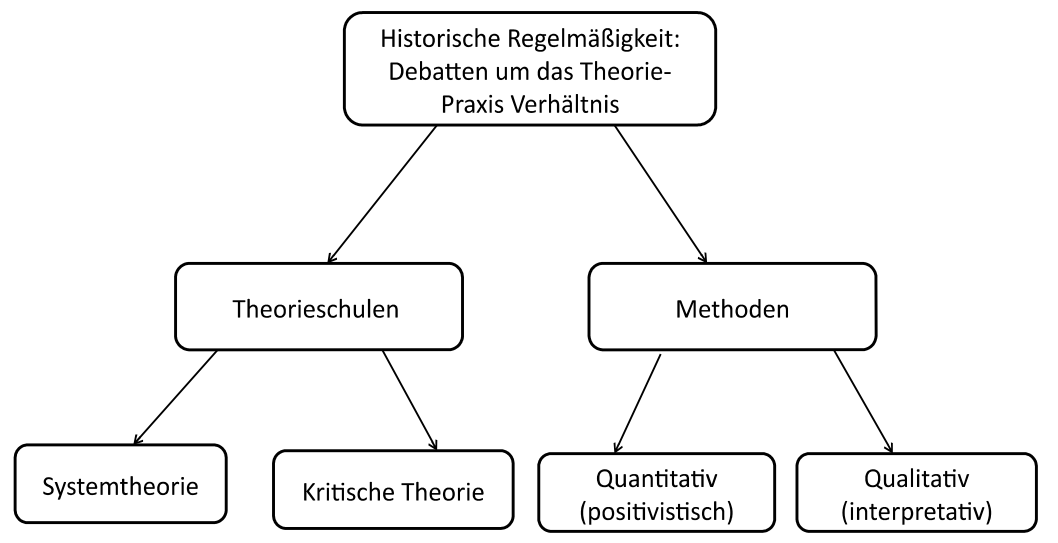

Die Fälle wurden so ausgewählt, dass sie maximal kontrastieren, jedoch innerhalb der Fallauswahl vergleichbar sind. Maximal kontrastierende Fälle im Sinne von Theorieschulen sind auf der einen Seite Repräsentant(inn)en der Systemtheorie, auf der anderen Seite Vertreter(innen) der kritischen Theorie. Hinsichtlich der Methoden wurden einerseits Interviewpartner(innen) ausgewählt, die ein stark quantitatives Paradigma vertreten. Auf der anderen Seite wurden Forschende befragt, die qualitative Forschung betreiben. Natürlich ist klar, dass sich Theorie- wie auch Methodenausrichtung überschneiden können. Es wurde deshalb angestrebt, Personen zu gewinnen, die sich durch eine überwiegende Orientierung in die eine oder die andere Richtung auszeichneten. Um Stereotypisierungen zu vermeiden, wird davon ausgegangen, dass auch innerhalb unterschiedlicher Theorieorientierungen unterschiedliche Ausprägungen bestehen.

\subsubsection{Die Datenbasis}

Wie bereits in der Grounded Theory konstatiert, bildet das theoretische Sampling eine Orientierung, die die Konversation zwischen Theorie und Empirie anregt. Dennoch kann das theoretische Sampling nur als grobe Richtschnur und nicht als strenges Vorgehen der Auswahl dienen, da es sonst dem Prinzip der Offenheit der Grounded Theory entgegenwirken würde. Zudem gilt auch hier, dass sich kaum eine Soziologin oder ein Soziologe einem einzigen Paradigma zuordnen 
lässt, wobei auch hier letztlich wichtig war, wie sich die Interviewpartner(innen) zum Zeitpunkt des Interviews positionierten.

Zudem ist klar, dass bei jeder Erhebung in einem gewissen Maß eine Selbstselektion stattfindet. So konnten eher Personen gewonnen werden, die sich für das Thema der Arbeit interessierten und möglicherweise selbst in der außersoziologischen Praxis aktiv waren. Forschende, die dieses Thema als Randthema wahrnahmen, waren meist nicht zu einem Interview bereit. Glücklicherweise konnten auch solche Personen gewonnen werden, die dem Thema kritisch gegenüberstanden, weshalb das Sample im Ganzen als ausgewogen gelten kann.

Die Interviews wurden in drei Erhebungswellen geführt. Jede Erhebungswelle wurde dazu genutzt, die empirischen und theoretischen Grundlagen der Arbeit miteinander in Verbindung zu setzen und die Auswahl der Interviewpartner(innen) ggf. zu modifizieren. Ebenso wurden ca. 25 Interviews in zwei Erhebungswellen in den USA, überwiegend in Ivy League-Universitäten, geführt. Diese wurden zwar nicht aktiv in die Analyse eingebunden, da dies den Rahmen der Arbeit sprengen würde. Sie beeinflussten jedoch das Hintergrundwissen um unterschiedliche nationale Soziologien und Formen der Grenzziehung. Insgesamt wurden sie als Bereicherung empfunden.

Bei der Auswahl der Interviewpartner(innen) wurde darauf geachtet, dass bei diesen bereits eine Hauptsozialisation im Wissenschaftssystem erfolgt war und eine akademische Karriere angestrebt bzw. bereits erreicht wurde. Die Interviews werden also auf der Ebene der Post-Docs, Juniorprofessor(inn)en und Professor(inn)en angesetzt. Es wurden insgesamt 21 Interviews mit Soziologinnen und Soziologen in Deutschland geführt. Davon hatten 14 Personen eine Professur unterschiedlicher Art inne (Vollprofessur, S-Professur, Juniorprofessur, Fachhochschulprofessur). Fünf Personen waren Senior-Researcher auf Post-DocNiveau. Zwei Interviewpartner(innen) befanden sich noch in der Qualifizierungsphase.

Die Interviews dauerten zwischen einer Stunde und eindreiviertel Stunden. Diese Unterschiede in der Dauer ergaben sich zunächst aus den zeitlichen Ressourcen der Interviewpartner(innen). Zudem waren es relativ offen geführte Interviews, in denen der Erzählfluss auch immer von der konkreten Person abhing. Das Hierarchiegefälle zwischen der Interviewenden und den Personen, die interviewt wurden, war meist groß, dennoch war die Gesprächsatmosphäre größtenteils kollegial geprägt. Da die Interviewerin aus der gleichen Disziplin stammte, bewegte sich das Verhältnis eher auf der Ebene der Co-Experten (Bogner et al., 2005b). In manchen Fällen wurde sogar eine Frage an die Interviewerin zurückgegeben, mit der Begründung, dass sie ja die Expertin für das Theorie-PraxisVerhältnis der Soziologie sei und nicht der bzw. die Befragte. In anderen Inter- 
views wurde die Interviewerin zusätzlich als normative Komplizin (Bogner et al., 2005b) in die Sinndeutungen der Interviewpartner einbezogen. In diesen Fällen war es besonders wichtig, die innere Distanz zu dem bzw. der Interviewten zu wahren. Diese Art der Interviews zeichnete sich dadurch aus, dass die Interviewten sich in einer sehr offenen und wenig formellen Sprache äußerten. Die Interviewerin wurde also gleichermaßen als Vertraute angesehen, vor der es keine Sprachetikette zu wahren galt. Dahingegen zeichneten sich andere Interviewpartner genau durch das Gegenteil aus: Namen von Kolleg(inn)en wurden nicht direkt genannt, Meinungen eher zurückhaltend geäußert. Hier wurde der Interviewerin möglicherweise die Rolle einer potenziellen Kritikerin (Bogner et al., 2005b) zugeschrieben. Dennoch wurden fast alle Interviews auf einer freundlichen und offenen Basis geführt.

Zudem spielten Geschlechter- und Altersunterschiede für die Gesprächsführung eine Rolle, ${ }^{18}$ wobei der Altersunterschied meist als dominanter wahrgenommen wurde als der Geschlechtsunterschied. So wurde mit Bemerkungen wie ,als ich in Ihrem Alter war" eindeutig auf Vergangenes hingewiesen und durch den Vergleich die Distanz zwischen dem eigenen Lebensabschnitt und dem der Interviewerin deutlich gemacht.

Weil die Interviewten als Soziolog(inn)en einen professionellen Blick auf die Interviews hatten, wurden sogenannte ,naive“ Fragen der Interviewerin nicht gleichfalls „naiv“ beantwortet, sondern aus der Perspektive des Wissenschaftlers bzw. der Wissenschaftlerin - es kommt also einmal mehr die Metaperspektive von Soziolog(inn)en auf die Soziologie zum Tragen. Die Metaperspektive bzw. disziplinäre Nähe zu der Interviewerin äußerte sich zudem in Fragen zum Leitfaden oder zur Auswertungstechnik der Arbeit. Dieses Oszillieren zwischen unterschiedlichen Ebenen in den Interviews stellt Momente der Selbstbeschreibung dar, die in der Auswertung in besonderer Weise berücksichtigt wurden.

Der Leitfaden zielte darauf ab, Semantiken und Rhetoriken der Selbstbeschreibung der Soziolog(inn)en zu erfassen und deren Grenzziehungen herauszukristallisieren. Der Leitfaden unterteilte sich in unterschiedliche Frageblöcke: Zunächst sollten die Forschenden beantworten, wie sie zur Soziologie und zu ihrem Forschungsthema gekommen waren. Darauf folgten in der Regel Ausführungen zur Biografie der Interviewpartner(innen), die mal ausführlicher und mal knapper ausfielen. Die Eingangsdarstellung wurde als besonders relevant angesehen. Ebenso wurde das Verständnis von „guter“ Soziologie erfasst und nachgeforscht, ob nach Meinung der Interviewten ein besonderer Auftrag der Soziologie in der Gesellschaft bestünde. Diese Frage wurde meist sehr kontrovers be-

18 Siehe dazu auch die Ausführungen von Lamont (1994). 
antwortet. Diese Momente waren für die Interviewführung wie auch für die Auswertung sehr erhellend, da gerade die Kontraste und die Reibungsmomente der diversen Selbstbeschreibungen Konflikte bei den Grenzziehungen deutlich machten. Um eine sogenannte „Leitfadenbürokratie“ zu vermeiden und narrative Momente zu erzeugen, wurden im Interview besonders Themen weiterverfolgt, die die Befragten von sich aus ansprachen. 